\title{
STRONG EQUALITY OF ROMAN AND PERFECT ROMAN DOMINATION IN TREES
}

\author{
Zehui Shao ${ }^{1}$, Saeed Kosari $^{1}$, Hadi Rahbani ${ }^{2}$, Mehdi Sharifzadeh $^{3}$ \\ AND Seyed Mahmoud SheikHoleslami ${ }^{2, *}$ (1)
}

\begin{abstract}
A Roman dominating function (RD-function) on a graph $G=(V, E)$ is a function $f$ : $V \longrightarrow\{0,1,2\}$ satisfying the condition that every vertex $u$ for which $f(u)=0$ is adjacent to at least one vertex $v$ for which $f(v)=2$. An Roman dominating function $f$ in a graph $G$ is perfect Roman dominating function (PRD-function) if every vertex $u$ with $f(u)=0$ is adjacent to exactly one vertex $v$ for which $f(v)=2$. The (perfect) Roman domination number $\gamma_{R}(G)\left(\gamma_{R}^{p}(G)\right)$ is the minimum weight of an (perfect) Roman dominating function on $G$. We say that $\gamma_{R}^{p}(G)$ strongly equals $\gamma_{R}(G)$, denoted by $\gamma_{R}^{p}(G) \equiv \gamma_{R}(G)$, if every RD-function on $G$ of minimum weight is a PRD-function. In this paper we show that for a given graph $G$, it is NP-hard to decide whether $\gamma_{R}^{p}(G)=\gamma_{R}(G)$ and also we provide a constructive characterization of trees $T$ with $\gamma_{R}^{p}(T) \equiv \gamma_{R}(T)$.
\end{abstract}

Mathematics Subject Classification. 05C69.

Received July 18, 2020. Accepted January 9, 2022.

\section{INTRODUCTION}

We consider finite, undirected, and simple graphs $G$ with vertex set $V=V(G)$ and edge set $E=E(G)$. The number of vertices of a graph $G$ is called the order of $G$ and is denoted by $n=n(G)$. For any vertex $v \in V(G)$, the open neighborhood of $v \in V$ is $N(v)=N_{G}(v)=\{u \in V \mid u v \in E\}$, and the degree of $v$, denoted by $\operatorname{deg}_{G}(v)$, is the cardinality of its open neighborhood. A leaf of a tree $T$ is a vertex of degree one, while a support vertex of $T$ is a vertex adjacent to a leaf. A strong support vertex is a support vertex adjacent to at least two leaves. In this paper, we denote by $L(x)$ the set of leaves adjacent to a support vertex $x$, and denote $\ell_{x}=|L(x)|$. A star is a non-trivial tree with at most one vertex which is not a leaf. We denote a star on $n \geq 2$ vertices by $K_{1, n-1}$. For $r, s \geq 1$, a double star, written $S(r, s)$, is a tree with exactly two non-leaf vertices, one of which has $r$ leaf neighbors, and the other has $s$ leaf neighbors. The length of a shortest $(u, v)$-path in a graph $G$ is the distance between $u$ and $v$, and is written $d_{G}(u, v)$ or $\operatorname{simply} d(u, v)$ if $G$ is clear from context. The diameter of $G$, written $\operatorname{diam}(G)$, is the maximum distance among all pairs of vertices in $G$.

Keywords. Perfect Roman dominating function, Roman dominating function.

1 Institute of Computing Science and Technology, Guangzhou University, Guangzhou 510006, P.R. China.

2 Department of Mathematics, Azarbaijan Shahid Madani University, Tabriz, Iran.

3 Department of Mathematics, Yasouj University, Yasouj, Iran.

*Corresponding author: s.m.sheikholeslami@azaruniv.ac.ir

(c) The authors. Published by EDP Sciences, ROADEF, SMAI 2022 
A rooted tree $T$ distinguishes one vertex $r$ called the root. For each vertex $v \neq r$ of $T$, the parent of $v$ is the neighbor of $v$ on the unique $(r, v)$-path, while a child of $v$ is any other neighbor of $v$. If $T$ is a rooted tree, then for any vertex $v$ we denote by $T_{v}$ the sub-rooted tree rooted at $v$.

A function $f: V(G) \rightarrow\{0,1,2\}$ is a Roman dominating function (or just RD-function) if every vertex $u$ for which $f(u)=0$ is adjacent to at least one vertex $v$ for which $f(v)=2$. The weight of an RD-function $f$ is $w(f)=f(V(G))=\sum_{u \in V(G)} f(u)$. The Roman domination number of a graph $G$, denoted by $\gamma_{R}(G)$, is the minimum weight of an RD-function on $G$. We refer to a $\gamma_{R}(G)$-function as an RD-function of $G$ with minimum weight. For more details on Roman domination and its variations we refer the reader to the recent book chapters and survey [5-9].

An RD-function $f=\left(V_{0}, V_{1}, V_{2}\right)$ is called a Perfect Roman dominating function (or just PRD-function) if very vertex $u$ with $f(u)=0$ is adjacent to exactly one vertex $v$ for which $f(v)=2$. The Perfect Roman domination number $\gamma_{R}^{P}(G)$ is the minimum weight of an PRD-function. We refer to a $\gamma_{R}^{P}(G)$-function as an PRD-function of $G$ with minimum weight. The concept of perfect Roman domination was introduced by Henning et al. [15] and has been studied in $[10,18]$.

Observe that $\gamma_{R}(G) \leq \gamma_{R}^{P}(G)$ for every graph $G$. Clearly, if $G$ is a graph with $\gamma_{R}(G)=\gamma_{R}^{P}(G)$, then every $\gamma_{R}^{P}(G)$-function is a $\gamma_{R}(G)$-function. However, not every $\gamma_{R}(G)$-function is an $\gamma_{R}^{P}(G)$-function even when $\gamma_{R}(G)=\gamma_{R}^{P}(G)$. For example consider the path $P_{5}$. We say that $\gamma_{R}^{P}(G)$ and $\gamma_{R}(G)$ are strongly equal, denoted by $\gamma_{R}(G) \equiv \gamma_{R}^{P}(G)$, if every $\gamma_{R}(G)$-function is an $\gamma_{R}^{P}(G)$-function.

In this paper we show that for a given graph $G$, it is NP-hard to decide whether $\gamma_{R}^{p}(G)=\gamma_{R}(G)$ and also in the next we provide a constructive characterization of trees $T$ with $\gamma_{R}^{p}(T) \equiv \gamma_{R}(T)$. Further examples of characterizations of tress can be found in $[1-4,13,16,17,19]$.

\section{Complexity}

In this section, we show that for a given graph $G$, it is NP-hard to decide whether $\gamma_{R}^{p}(G)=\gamma_{R}(G)$. Consider the following decision problem.

$\gamma_{R}^{p}(G)=\gamma_{R}(G)$ Problem

Instance: Graph $G=(V, E)$.

Question: Does for graph $G, \gamma_{R}^{p}(G)=\gamma_{R}(G)$ ?

Our reduction is from the following problem.

EXACT 3-COVER $(X 3 C)$

Instance: A finite set $X$ with $|X|=3 q$ and a collection $C$ of 3-element subsets of $X$.

Question: Is there a subcollection $C^{\prime}$ of $C$ such that every element of $X$ appears in exactly one element of $C^{\prime}$ ?

It is well known that X3C is NP-complete [12]. We show that equality of Roman and perfect Roman domination problem is NP-complete by reducing from EXACT 3-COVER problem.

Theorem 2.1. For a given graph $G$, it is $N P$-hard to decide whether $\gamma_{R}^{p}(G)=\gamma_{R}(G)$.

Proof. Clearly, the $\gamma_{R}^{p}(G)=\gamma_{R}(G)$ problem is in $N P$, since it is easy to verify that for a given graph $G$, $\gamma_{R}^{p}(G)=\gamma_{R}(G)$ in polynomial time. Now let us show how to transform any instance $X, C$ of $X 3 C$ into an instance $G$ of $\gamma_{R}^{p}(G)=\gamma_{R}(G)$ problems so that one of them has a solution if and only if the other has a solution. Let $X=\left\{x_{1}, x_{2}, \ldots, x_{3 q}\right\}$ and $C=\left\{C_{1}, C_{2}, \ldots, C_{t}\right\}$ be an arbitrary instance of $X 3 C$. We join each vertex $x_{i} \in X$ with the leaf $y_{i}$ of a stare $K_{1,4}$ with chenter $w_{i}$. For each $C_{j} \in C$, we build a star $K_{1,3}$ centered at $z_{j}$ for which one leaf is labeled $c_{j}$. Now to obtain a graph $G$, we add edges $c_{j} x_{i}$ if $x_{i} \in C_{j}$ and for any $i=3 l+2$ that $l \geq 0$ we add edges $x_{i} x_{i-1}, x_{i} x_{i+1}$ and $x_{i-1} x_{i+1}$. Figure 1 shows an example of graphs $G$. Set $k=2 t+8 q$.

Claim 2.2. $\gamma_{R}(G)=k$. 


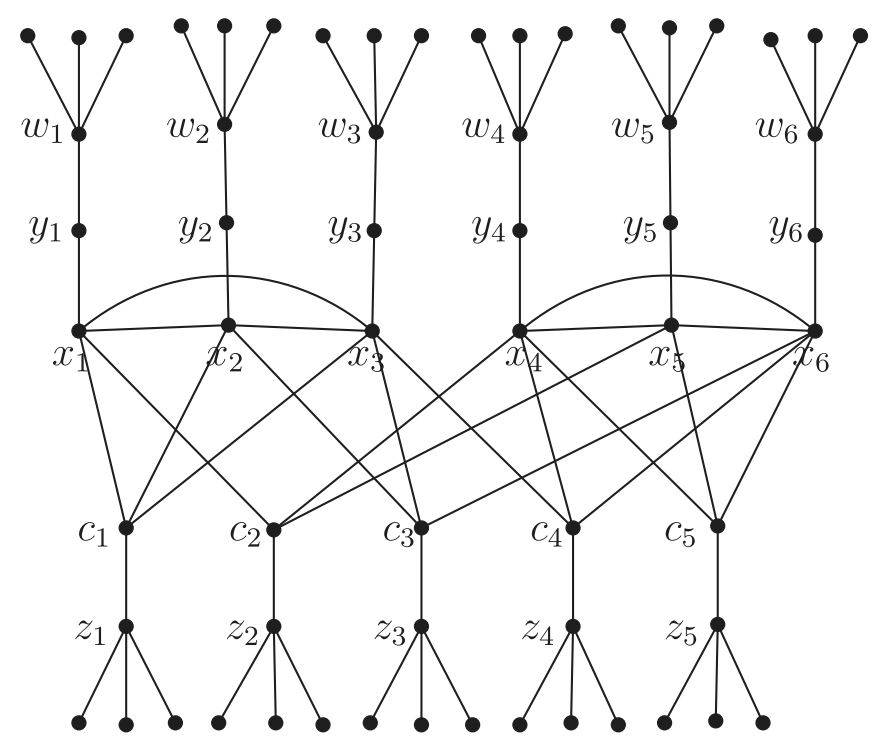

Figure 1. The graph $G$ in the proof of Theorem 2.1.

Proof. Let $B$ be set of all $x_{i}$ such that $i=3 l+2$ for $l \geq 0$ and $D=B \cup \bigcup_{i=1}^{i=3 q}\left\{w_{i}\right\} \cup \bigcup_{j=1}^{i=t}\left\{z_{j}\right\}$. Then, clearly function $g=(V-D, \emptyset, D)$ is a RD-function and so $\gamma_{R}(G) \leq w(g)=8 q+2 t=k$. Now assume that $f=\left(V_{0}, V_{1}, V_{2}\right)$ is a $\gamma_{R}(G)$-the function. Then, we can see that $\bigcup_{i=1}^{i=3 q}\left\{w_{i}\right\} \cup \bigcup_{j=1}^{i=t}\left\{z_{j}\right\} \subseteq V_{2}$ and for every $j \in\{1,2, \ldots, t\}, f\left(c_{j}\right)=0$ or $f\left(c_{j}\right)=2$. Let $R=\left\{j: c_{j} \in V_{2}\right\}$ and $|R|=r$. Also let $M=\left\{i: x_{i} \in V_{2}\right\}$, $N=\left\{i: x_{i} \in V_{1}\right\},|M|=m$ and $|N|=n$. Then $\gamma_{R}(G)=w(f)=2 t+6 q+2 r+2 m+n$. We first assume that $r+m<q$. Since each $c_{j} \in R$ and $x_{i} \in M$ has exactly three neighbors in $\left\{x_{1}, x_{2}, \ldots, x_{3 q}\right\}$, we deduce that $|N| \geq 3 q-3 r-3 m$. Hence

$$
\begin{aligned}
\gamma_{R}(G) & =w(f) \geq 2 t+6 q+2 r+2 m+n \\
& \geq 2 t+6 q+2 r+2 m+3 q-3 r-3 m \\
& =2 t+8 q+(q-r-m) \\
& >2 t+8 q \\
& \geq \gamma_{R}(G),
\end{aligned}
$$

a contradiction. Thus we may assume that $r+m \geq q$. Then

$$
\begin{aligned}
\gamma_{R}(G) & =w(f) \\
& =2 t+6 q+2 r+2 m+n \\
& =2 t+8 q+n \\
& \geq 2 t+8 q .
\end{aligned}
$$

Consequently, $\gamma_{R}(G)=2 t+8 q$.

Now assme that $h$ is a $\gamma_{R}^{p}(G)$-function with weight $k$. Clearly, each star needs a weight of at least 2, and so we may assume that $h\left(z_{j}\right)=h\left(w_{i}\right)=2$ and all its leaves are assigned 0 . Since $y_{i} w_{i} \in E(G)$, it follows that each vertex $w_{i}$ may be assigned the value 0 . If there exist $i$ such that $h\left(x_{i}\right) \neq 0$, then $h\left(x_{i}\right)=1$, since $h$ is a $\gamma_{R}^{p}(G)$ function. Let $S=\left\{i: x_{i} \in V_{1}\right\}$ and $|S|=s$. Then for each $i \notin S$, there exist a vertex $c_{j}$ for some $j=1,2, \ldots, t$ such that $x_{i} \in C_{j}$ and $c_{j} \in V_{2}$. Let $p$ be the number of $c_{j}$ 's belonging to $V_{2}$. Then $s+6 q+2 p+2 t \leq 2 t+8 q$ and so $s+2 p \leq 2 q$. On the other hand, since each $c_{j}$ has exactly three neighbors in $\left\{x_{1}, x_{2}, \ldots, x_{3 q}\right\}$, we have 
$3 p \geq 3 q-s$. Combining these two inequalities, we arrive at $p=q$ and $r=0$. Consequently, $C^{\prime}=\left\{C_{j}: c_{j} \in S\right\}$ is an exact cover for $C$.

Conversely, suppose that the instance $X, C$ of $X 3 C$ has a solution $C^{\prime}$. We construct a perfect Roman dominating function $f$ on $G$ of weight $k$. For every $C_{j}$, assign the value 2 to $c_{j}$ if $C_{j} \in C^{\prime}, 0$ if $C_{j} \notin C^{\prime}$, assign 2 to every $z_{j}$ and every $w_{i}$ and 0 to the remaining vertices of $G$. Thereby since $C^{\prime}$ exists, its cardinality is precisely $q$, the number of $c_{j}$ 's with weight 2 is $q$, having disjoint neighborhoods in $\left\{x_{1}, x_{2}, \ldots, x_{3 q}\right\}$. Hence, it is straightforward to see that $f$ is a perfect Roman dominating function with weight $k$. Hence we obtain that $\gamma_{R}^{p}(G)=\gamma_{R}(G)$ if and only if there a subcollection $C^{\prime}$ of $C$ such that every element of $X$ appears in exactly one element of $C^{\prime}$.

\section{Constructive Characterization of Strong Equality}

We make use of the following.

Proposition $3.1([11])$. Let $f=\left(V_{0}, V_{1}, V_{2}\right)$ be a $\gamma_{R}(G)$-function. Then

(1) The subgraph induced by $V_{1}$ has maximum degree one.

(2) No edge of $G$ joins $V_{1}$ to $V_{2}$.

We begin with the following lemmas.

Lemma 3.2. Let $G$ be a connected graph of order $n \geq 3$. If $\gamma_{R}^{p}(T) \equiv \gamma_{R}(T)$, then for every $\gamma_{R}(G)$-function $f=\left(V_{0}, V_{1}, V_{2}\right), V_{1}$ is independent.

Proof. Suppose, to the contrary, $V_{1}$ is not independent. By Proposition 3.1, $G\left[V_{1}\right]$ has an edge $u v$. Since $n \geq 3$, we may assume, without loss of generality, that $\operatorname{deg}(u)>1$. Let $w$ be the neighbor of $u$ different from $v$. Then $f(w)=0$, and so there is a vertex $r \in V_{2}$ such that $r \in N(w)$. Then reassigning to $u$ the weight 2, to $v$ the weight 0 and leaving all other weights unchanged produces a new $\gamma_{R}(T)$-function that is not a PRD-function, contradicting the fact that $\gamma_{R}^{p}(T) \equiv \gamma_{R}(T)$.

Now we present a constructive characterization of trees $T$ with $\gamma_{R}^{p}(T) \equiv \gamma_{R}(T)$. For this purpose, we define a family of trees as follows. Let $\mathcal{F}$ be the collection of trees $T$ that can be obtained from a sequence $T_{1}, T_{2}, \ldots, T_{k}=$ $T(k \geq 1)$ of trees, where $T_{1} \in\left\{P_{2}, P_{3}\right\}$ and $T=T_{k}$. Further, if $k \geq 1$, then for each $i \in[k]$, the tree $T_{i}$ can be obtained from the tree $T^{\prime}=T_{i-1}$ by one of the following eleven operations $\varphi_{1}, \varphi_{2}, \ldots, \varphi_{11}$ defined below and illustrated in Figure 2.

- Operation $\varphi_{1}$ : add a new vertex $u$ to $T^{\prime}$ and join it to a strong support vertex $v$ of $T^{\prime}$.

- Operation $\varphi_{2}$ : add a star $K_{1,3}$ and join a leaf $v$ of star to a vertex $u$ of $T^{\prime}$ that cannot be assigned the value 2 under any $\gamma_{R}$-function of $T^{\prime}$.

- Operation $\varphi_{3}$ : add a double star $S(2,1)$, and join the support vertex $v$ of the double star with degree two

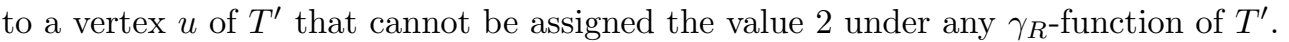

- Operation $\varphi_{4}$ : add a star $K_{1,2}$ with central vertex $v$, and join the vertex $v$ to vertex $u$ of $T^{\prime}$ that is assigned the value 2 by every $\gamma_{R}$-function of $T^{\prime}$ and is adjacent to a strong support vertex of degree 3 in $T^{\prime}$.

- Operation $\varphi_{5}$ : add a star $K_{1,2}$ with central vertex $v$, and join the vertex $v$ to vertex $u$ of $T^{\prime}$ that is assigned the value 2 by every $\gamma_{R^{-}}$function of $T^{\prime}$ and is adjacent to a weak support vertex of degree 2 in $T^{\prime}$.

- Operation $\varphi_{6}$ : add a star $K_{1,2}$ centred at $v$, and join the vertex $v$ to stong support vertex $u$ of $T^{\prime}$ with degree three, that is assigned the value 2 by every $\gamma_{R}$-function of $T^{\prime}$.

- Operation $\varphi_{7}$ : add a path $P_{2}: v w$ and join the vertex $v$ of the path to a strong support vertex $u$ of $T^{\prime}$.

- Operation $\varphi_{8}$ : add a new vertex $v$ to $T^{\prime}$ and join it to leaf neighbors $u$ of a strong support vertex $w$ of $T^{\prime}$ with exactly two neighbors leaves in $T^{\prime}$.

- Operation $\varphi_{9}$ : add a new vertex $v$ to $T^{\prime}$ and join $v$ to a leaf $u$ of $T^{\prime}$ such that the vertex $w$ that $\{w\}=N(u)$ has at least two neighbors which are weak support vertices with degree two. 

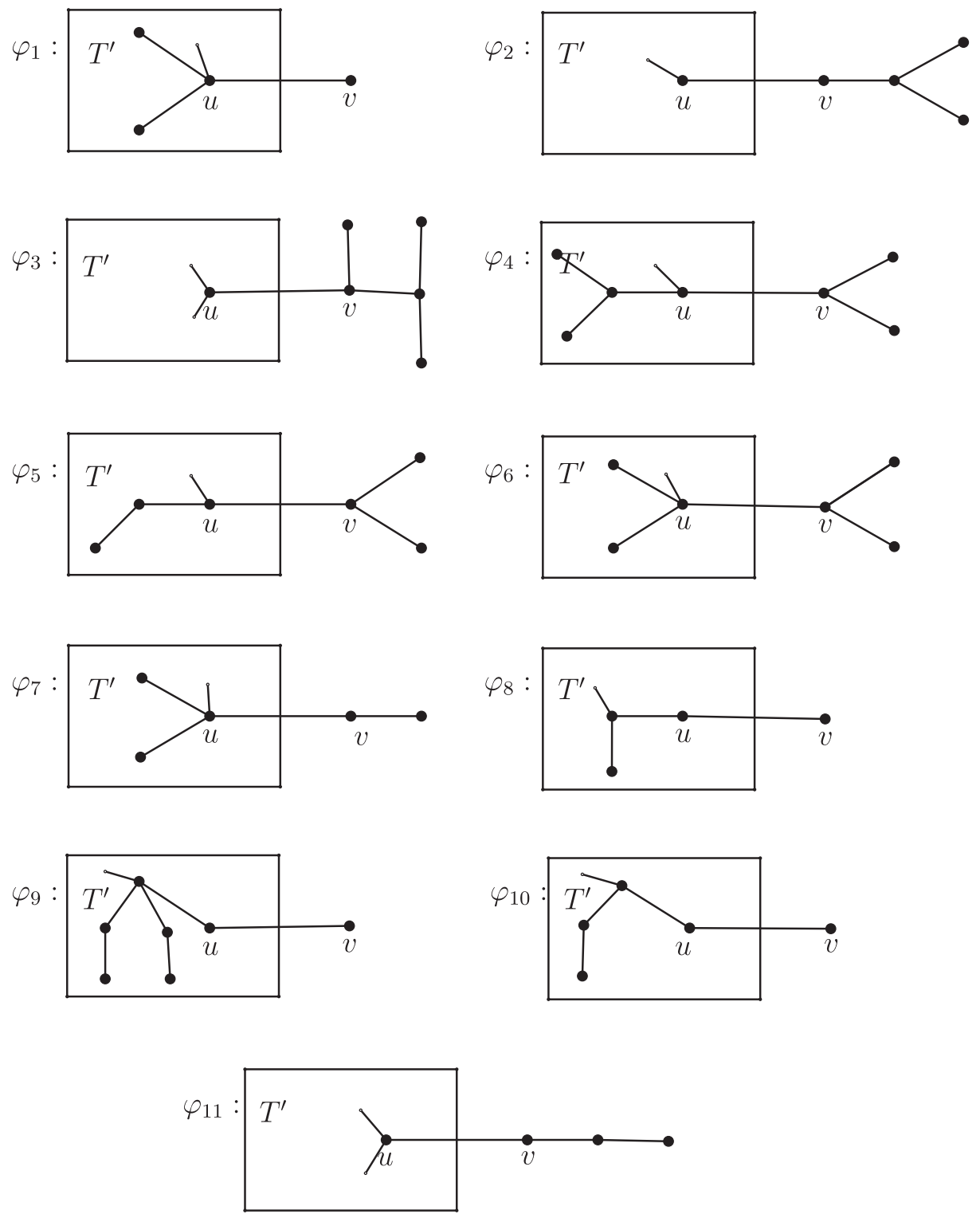

FiguRE 2. The operations $\varphi_{1}, \varphi_{2}, \ldots, \varphi_{11}$.

- Operation $\varphi_{10}$ : add a new vertex $v$ to $T^{\prime}$ and join $v$ to a leaf $u$ of $T^{\prime}$ such that the vertex $w$ that $\{w\}=N(u)$ has exactly one neighbor weak support vertex with degree two and is assigned the value 2 by every $\gamma_{R}$-function of $T^{\prime}$.

- Operation $\varphi_{11}$ : add a path $P_{3}: v x y$ and join leaf $v$ of the path to vertex $u$ of $T^{\prime}$ that cannot be assigned the value 2 under any $\gamma_{R^{-}}$function of $T^{\prime}$.

We show next that for every tree $T$ in the family $\mathcal{F}, \gamma_{R}^{p}(T)$ strongly equals $\gamma_{R}(T)$. 
Lemma 3.3. If $T$ is a tree in the family $\mathcal{F}$, then $\gamma_{R}^{p}(T) \equiv \gamma_{R}(T)$.

Proof. We proceed by induction on the order $n \geq 2$ of a tree $T \in \mathcal{T}$. If $n \in\{2,3\}$, then $T \in\left\{P_{2}, P_{3}\right\}$ and clearly $\gamma_{R}^{p}(T) \equiv \gamma_{R}(T)$. Suppose that $n \geq 4$ and that for every tree in $\mathcal{F}$ of order $n^{\prime}$, where $4 \leq n^{\prime}<n$, $\gamma_{R}^{p}(T) \equiv \gamma_{R}(T)$. Let $T \in \mathcal{F}$ have order $n$. Thus, $T$ can be obtained from a sequence of trees $T_{1}, \ldots, T_{k}$, where $k \geq 1, T_{1} \in\left\{P_{2}, P_{3}\right\}, T=T_{k}$, and for each $i \leq k-1$, the tree $T_{i+1}$ can be obtained from the tree $T_{i}$ by one of the eleven operations $\varphi_{1}, \varphi_{2}, \ldots, \varphi_{11}$. Let $T^{\prime}=T_{k-1}$, and so $T^{\prime} \in \mathcal{T}$ has order less than $n$. Applying the inductive hypothesis to $T^{\prime}, \gamma_{R}^{p}\left(T^{\prime}\right) \equiv \gamma_{R}\left(T^{\prime}\right)$. Let $u$ be the attacher of $T^{\prime}$ and $v$ the link vertex of $T$ used to construct the tree $T$ from the tree $T^{\prime}$.

Let $f$ be such a $\gamma_{R}$-function of $T$ chosen so that the sum of the values assigned to all leaves under $f$ is minimum. Let $f^{\prime}$ be the restriction of the function $f$ to the tree $T^{\prime}$. Thus, $f^{\prime}(w)=f(w)$ for every vertex $z \in V\left(T^{\prime}\right)$. We consider eleven cases, depending on which operation is used to construct the tree $T$ from $T^{\prime}$.

Case 1. $T$ is obtained from $T^{\prime}$ by Operation $\varphi_{1}$.

Let $g^{\prime}$ be a $\gamma_{R}^{P}\left(T^{\prime}\right)$-function. Since $u$ is a strong support vertex of $T^{\prime}$ and $\gamma_{R}\left(T^{\prime}\right) \equiv \gamma_{R}^{P}\left(T^{\prime}\right)$, we can assume that $g^{\prime}(u)=2$. Then $g^{\prime}$ can be extended to a PRD-function $g$ on $T$ by assigning the weight 0 to $v$. The resulting PRD-function $g$ has weight $w(g)=w\left(g^{\prime}\right)=\gamma_{R}^{P}\left(T^{\prime}\right)$ and so by the statement above and inductive hypothesis, we obtain

$$
\gamma_{R}(T) \leq \gamma_{R}^{P}(T) \leq w(g)=w\left(g^{\prime}\right)=\gamma_{R}^{P}\left(T^{\prime}\right)=\gamma_{R}\left(T^{\prime}\right) .
$$

Conversely, the vertex $u$ is a strong support vertex of $T$ with $\ell_{u} \geq 3$ and so $f(u)=2$ and $f(v)=0$. Then, $f^{\prime}$ is a RD-function on $T^{\prime}$ of weight $\gamma_{R}(T)$, from which we deduce that $\gamma_{R}\left(T^{\prime}\right) \leq \gamma_{R}(T)$. Consequently, we must have equalities throughout the inequality chain (3.1). In particular, $\gamma_{R}(T)=\gamma_{R}^{P}(T)$.

Now we show that $\gamma_{R}(T) \equiv \gamma_{R}^{P}(T)$. Suppose, to the contrary, $\gamma_{R}(T) \not \equiv \gamma_{R}^{P}(T)$. Then there is a $\gamma_{R}(T)$ function $g$ such that $g$ is not a PRD-function. Since $u$ is a strong support vertex, $g(u)=2$ and $g(v)=0$. Thus, $g$ restricted to $V\left(T^{\prime}\right)$ is a $\gamma_{R}$-function on $T^{\prime}$ that it is not a PRD-function, contradicting the fact that $\gamma_{R}^{p}\left(T^{\prime}\right) \equiv \gamma_{R}\left(T^{\prime}\right)$. Hence $\gamma_{R}(T) \equiv \gamma_{R}^{P}(T)$.

Case 2. $T$ is obtained from $T^{\prime}$ by Operation $\varphi_{2}$.

Let $v_{1}$ be the central vertex of the added star $K_{1,3}$ when constructing $T$ from $T^{\prime}$. Let $g^{\prime}$ be a $\gamma_{R}^{P}\left(T^{\prime}\right)$-function. By assumption, $g^{\prime}$ is a $\gamma_{R}^{P}\left(T^{\prime}\right)$-function and $g^{\prime}(u) \neq 2$. Then $g^{\prime}$ can be extended to a PRD-function $g$ on $T$ by assigning to $v_{1}$ the value 2 and to its neighbors the weight 0 , implying that $\gamma_{R}^{P}(T) \leq w(g)=w\left(g^{\prime}\right)+2=$ $\gamma_{R}^{P}\left(T^{\prime}\right)+2$. Hence by the statement above and inductive hypothesis, we obtain

$$
\gamma_{R}(T) \leq \gamma_{R}^{P}(T) \leq w(g)=w\left(g^{\prime}\right)+2=\gamma_{R}^{P}\left(T^{\prime}\right)+2=\gamma_{R}\left(T^{\prime}\right)+2 .
$$

Conversely, the vertex $v_{1}$ is a strong support vertex of $T$ and so we can assume that $f\left(v_{1}\right)=2$ and $f(v)=0$. Thus, $f(v)=0$ implies $f^{\prime}$ is a RD-function on $T^{\prime}$, from which we deduce that $\gamma_{R}\left(T^{\prime}\right) \leq \gamma_{R}(T)-2$. Consequently, we must have equalities throughout the inequality chain (3.2). In particular, $\gamma_{R}(T)=\gamma_{R}^{P}(T)$. Now we show that $\gamma_{R}(T) \equiv \gamma_{R}^{P}(T)$. Suppose, to the contrary, $\gamma_{R}(T) \not \equiv \gamma_{R}^{P}(T)$. Then there is a $\gamma_{R}(T)$-function $g$ such that $g$ is not a PRD-function. Since $v_{1}$ is a strong support vertex, we can assume that $g\left(v_{1}\right)=2$ and $g(v)=0$. Thus, $g$ restricted to $V\left(T^{\prime}\right)$ is a $\gamma_{R}$-function on $T^{\prime}$ that it is not a PRD-function, contradicting the fact that $\gamma_{R}^{p}\left(T^{\prime}\right) \equiv \gamma_{R}\left(T^{\prime}\right)$. Hence $\gamma_{R}(T) \equiv \gamma_{R}^{P}(T)$.

Case 3. $T$ is obtained from $T^{\prime}$ by Operation $\varphi_{3}$.

Let $v$ and $w$ be the two central vertices of the added double star $S(2,1)$, where the link vertex $v$ is adjacent to leaf $x$. Let $g^{\prime}$ be a $\gamma_{R}^{P}\left(T^{\prime}\right)$-function. By assumption, $g^{\prime}(u) \neq 2$. Then $g^{\prime}$ can be extended to a PRD-function $g$ on $T$ by assigning to $x$ the value 1 , to $w$ the value 2 and to its neighbors the weight 0 , implying that $\gamma_{R}^{P}(T) \leq w(g)=w\left(g^{\prime}\right)+3=\gamma_{R}^{P}\left(T^{\prime}\right)+3$. Hence by the inductive hypothesis, we obtain

$$
\gamma_{R}(T) \leq \gamma_{R}^{P}(T) \leq w(g)=w\left(g^{\prime}\right)+3=\gamma_{R}^{P}\left(T^{\prime}\right)+3=\gamma_{R}\left(T^{\prime}\right)+3 .
$$

On the other hand, since the vertex $w$ is a strong support vertex of $T$, we can assume that $f(w)=2$. Without loss of generality, we assume that $f(v)=0$ and $f(x)=1$. Thus, $f^{\prime}$ is a RD-function on $T^{\prime}$, from 
which we deduce that $\gamma_{R}\left(T^{\prime}\right) \leq \gamma_{R}(T)-3$. Consequently, we must have equalities throughout the inequality chain (3.3). In particular, $\gamma_{R}(T)=\gamma_{R}^{P}(T)$.

Next we show that $\gamma_{R}(T) \equiv \gamma_{R}^{P}(T)$. Suppose, to the contrary, $\gamma_{R}(T) \not \equiv \gamma_{R}^{P}(T)$. Then there is a $\gamma_{R}(T)$-function $g$ such that $g$ is not a PRD-function. Since $w$ is a strong support vertex, we can assume that $g(w)=2$. Clearly $g(v)=2$ or $g(v)=0$. We first assume that $g(v)=2$, then without loss of generality we can assume that $g(u)=0$ and for $y \in N(u)-\{v\}, f(y) \neq 2$, for otherwise we can reassign to $v$ the weight 0 and to $x$ the weight 1 to produce a RD-function of smaller weight than $w(g)=\gamma_{R}(T)$, a contradiction. Then the function $h: V\left(T^{\prime}\right) \longrightarrow\{0,1,2\}$ with $h(u)=1$ and for $z \neq u, h(z)=g(z)$ is a $\gamma_{R}\left(T^{\prime}\right)$-function that it is not a PRD-function, contradicting $\gamma_{R}^{p}\left(T^{\prime}\right) \equiv \gamma_{R}\left(T^{\prime}\right)$. Now assume that $g(v)=0$, then $\left.g\right|_{T^{\prime}}$ is a $\gamma_{R}^{P}\left(T^{\prime}\right)$-function and so by our earlier assumptions $\left.g\right|_{T^{\prime}}(u) \neq 2$. Then $\left.g\right|_{T^{\prime}}$ is not a PRD-function, contradicting the fact that, $\gamma_{R}^{p}\left(T^{\prime}\right) \equiv \gamma_{R}\left(T^{\prime}\right)$. Hence in two cases, $\gamma_{R}(T) \equiv \gamma_{R}^{P}(T)$.

Case 4. $T$ is obtained from $T^{\prime}$ by Operation $\varphi_{4}$.

Let $w$ be the strong support vertex of degree 3 in $T^{\prime}$ adjacent to $u$. Let $g^{\prime}$ be a $\gamma_{R}^{P}\left(T^{\prime}\right)$-function. By assumption, $g^{\prime}(u)=2$. Then $g^{\prime}$ can be extended to a PRD-function $g$ on $T$ by assigning to $v$ the value 2 and to its leaf neighbors the weight 0 . Hence, $\gamma_{R}^{P}(T) \leq w(g)=w\left(g^{\prime}\right)+2=\gamma_{R}^{P}\left(T^{\prime}\right)+2$ and so by the inductive hypothesis, we obtain

$$
\gamma_{R}(T) \leq \gamma_{R}^{P}(T) \leq w(g)=w\left(g^{\prime}\right)+2=\gamma_{R}^{P}\left(T^{\prime}\right)+2=\gamma_{R}\left(T^{\prime}\right)+2 .
$$

On the other hand, $v$ and $w$ are two strong support vertices of $T$ and so we can assume that $f(v)=f(w)=2$. Thus, $f^{\prime}$ is a RD-function on $T^{\prime}$, from which we deduce that $\gamma_{R}\left(T^{\prime}\right) \leq \gamma_{R}(T)-2$. Consequently, we must have equalities throughout the inequality chain (3.4). In particular, $\gamma_{R}(T)=\gamma_{R}^{P}(T)$.

Next we show that $\gamma_{R}(T) \equiv \gamma_{R}^{P}(T)$. Suppose, to the contrary, $\gamma_{R}(T) \not \equiv \gamma_{R}^{P}(T)$. Then there is a $\gamma_{R}(T)$ function $g$ such that $g$ is not a PRD-function. Since $w$ and $v$ are two strong support vertex, we can assume that $g(w)=g(v)=2$. Then $g^{\prime}=\left.g\right|_{T^{\prime}}$ is $\gamma_{R}\left(T^{\prime}\right)$-function and so by our earlier assumption $g^{\prime}(u)=2$. Therefore, $g^{\prime}$ is not a PRD-function, contradicting $\gamma_{R}^{p}\left(T^{\prime}\right) \equiv \gamma_{R}\left(T^{\prime}\right)$. Hence $\gamma_{R}(T) \equiv \gamma_{R}^{P}(T)$.

Case 5. $T$ is obtained from $T^{\prime}$ by Operation $\varphi_{5}$.

Let $w$ be the weak support vertex of degree 2 in $T^{\prime}$ adjacent to $u$. Let $g^{\prime}$ be a $\gamma_{R}^{P}\left(T^{\prime}\right)$-function. By assumption, $g^{\prime}(u)=2$. Then $g^{\prime}$ can be extended to a PRD-function $g$ on $T$ by assigning to $v$ the value 2 and to its leaf neighbors the weight 0 . Hence $\gamma_{R}^{P}(T) \leq w(g)=w\left(g^{\prime}\right)+2=\gamma_{R}^{P}\left(T^{\prime}\right)+2$ and so by the inductive hypothesis, we obtain

$$
\gamma_{R}(T) \leq \gamma_{R}^{P}(T) \leq w(g)=w\left(g^{\prime}\right)+2=\gamma_{R}^{P}\left(T^{\prime}\right)+2=\gamma_{R}\left(T^{\prime}\right)+2 .
$$

On the other hand, $v$ is a strong support vertices of $T$ and so we can assume that $f(v)=2$. Clearly $f(u) \neq 0$ or we can assume that $f(w)=2$. In two cases, $f^{\prime}$ is a RD-function on $T^{\prime}$, from which we deduce that $\gamma_{R}\left(T^{\prime}\right) \leq \gamma_{R}(T)-2$. Consequently, we must have equalities throughout the inequality chain (3.5). In particular, $\gamma_{R}(T)=\gamma_{R}^{P}(T)$.

Next we show that $\gamma_{R}(T) \equiv \gamma_{R}^{P}(T)$. Suppose, to the contrary, $\gamma_{R}(T) \not \equiv \gamma_{R}^{P}(T)$. Then there is a $\gamma_{R}(T)$-function $g$ such that $g$ is not a PRD-function. Since $v$ is a strong support vertex, we can assume that $g(v)=2$. Clearly, $g(u) \neq 0$ or we can assume that $g(w)=2$. In two cases $g^{\prime}=\left.g\right|_{T^{\prime}}$ is $\gamma_{R}\left(T^{\prime}\right)$-function and so by our earlier assumption $g^{\prime}(u)=2$. Therefore, $g^{\prime}$ is not a PRD-function, contradicting the fact that $\gamma_{R}^{p}\left(T^{\prime}\right) \equiv \gamma_{R}\left(T^{\prime}\right)$. Hence $\gamma_{R}(T) \equiv \gamma_{R}^{P}(T)$.

Case 6. $T$ is obtained from $T^{\prime}$ by Operation $\varphi_{6}$.

Let $g^{\prime}$ be a $\gamma_{R}^{P}\left(T^{\prime}\right)$-function. By assumption, $g^{\prime}(u)=2$. Then $g^{\prime}$ can be extended to a PRD-function $g$ on $T$ by assigning to $v$ the value 2 and to its leaf neighbors the weight 0 . Hence $\gamma_{R}^{P}(T) \leq w(g)=w\left(g^{\prime}\right)+2=\gamma_{R}^{P}\left(T^{\prime}\right)+2$ and so by the inductive hypothesis, we obtain

$$
\gamma_{R}(T) \leq \gamma_{R}^{P}(T) \leq w(g)=w\left(g^{\prime}\right)+2=\gamma_{R}^{P}\left(T^{\prime}\right)+2=\gamma_{R}\left(T^{\prime}\right)+2 .
$$

On the other hand, since $u$ and $v$ are strong support vertices of $T$, we can assume that $f(v)=f(u)=2$. Then $f^{\prime}$ is a RD-function on $T^{\prime}$, from which we deduce that $\gamma_{R}\left(T^{\prime}\right) \leq \gamma_{R}(T)-2$. Consequently, we must have equalities throughout the inequality chain (3.6). In particular, $\gamma_{R}(T)=\gamma_{R}^{P}(T)$. 
Now we show that $\gamma_{R}(T) \equiv \gamma_{R}^{P}(T)$. Suppose, to the contrary, $\gamma_{R}(T) \not \equiv \gamma_{R}^{P}(T)$. Then there is a $\gamma_{R}(T)$-function $g$ such that $g$ is not a PRD-function. Since $u$ and $v$ are two strong support vertices of $T$, we can assume that $g(v)=g(u)=2$. Hence $\left.g\right|_{T^{\prime}}$ is $\gamma_{R}\left(T^{\prime}\right)$-function that is not a PRD-function, contradicting the fact that $\gamma_{R}^{p}\left(T^{\prime}\right) \equiv \gamma_{R}\left(T^{\prime}\right)$. Hence $\gamma_{R}(T) \equiv \gamma_{R}^{P}(T)$.

Case 7. $T$ is obtained from $T^{\prime}$ by Operation $\varphi_{7}$.

Let $w$ be leaf neighbors of $v$ and $g^{\prime}$ be a $\gamma_{R}^{P}\left(T^{\prime}\right)$-function. Since, $\gamma_{R}^{p}\left(T^{\prime}\right) \equiv \gamma_{R}\left(T^{\prime}\right)$, we can assume that $g^{\prime}(u)=2$. Then $g^{\prime}$ can be extended to a PRD-function $g$ on $T$ by assigning to $v$ the value 0 and to its leaf neighbor the weight 1 . Hence $\gamma_{R}^{P}(T) \leq w(g)=w\left(g^{\prime}\right)+1=\gamma_{R}^{P}\left(T^{\prime}\right)+1$ and so by the inductive hypothesis, we obtain

$$
\gamma_{R}(T) \leq \gamma_{R}^{P}(T) \leq w(g)=w\left(g^{\prime}\right)+1=\gamma_{R}^{P}\left(T^{\prime}\right)+1=\gamma_{R}\left(T^{\prime}\right)+1 .
$$

On the other hand, we can assume that $f(u)=2, f(v)=0$ and $f(w)=1$. Then $f^{\prime}$ is a RD-function on $T^{\prime}$, from which we deduce that $\gamma_{R}\left(T^{\prime}\right) \leq \gamma_{R}(T)-1$ and as above we have $\gamma_{R}(T)=\gamma_{R}^{P}(T)$.

Next we show that $\gamma_{R}(T) \equiv \gamma_{R}^{P}(T)$. Suppose, to the contrary, $\gamma_{R}(T) \not \equiv \gamma_{R}^{P}(T)$. Then there is a $\gamma_{R}(T)$-function $g$ such that $g$ is not a PRD-function. Since $u$ is a strong support vertex of $T$, we can assume that $g(u)=2$. Hence $\left.g\right|_{T^{\prime}}$ is $\gamma_{R}\left(T^{\prime}\right)$-function that is not a PRD-function, contradicting the fact that $\gamma_{R}^{p}\left(T^{\prime}\right) \equiv \gamma_{R}\left(T^{\prime}\right)$. Hence $\gamma_{R}(T) \equiv \gamma_{R}^{P}(T)$.

Case 8. $T$ is obtained from $T^{\prime}$ by Operation $\varphi_{8}$.

Let $z$ be leaf neighbor of $w$ other than $u$ and $g^{\prime}$ be a $\gamma_{R}^{P}\left(T^{\prime}\right)$-function. Then $g^{\prime}$ can be extended to a PRDfunction $g$ on $T$ by assigning to $v$ the value 1 . Hence $\gamma_{R}^{P}(T) \leq w(g)=w\left(g^{\prime}\right)+1=\gamma_{R}^{P}\left(T^{\prime}\right)+1$ and so by the inductive hypothesis, we obtain

$$
\gamma_{R}(T) \leq \gamma_{R}^{P}(T) \leq w(g)=w\left(g^{\prime}\right)+1=\gamma_{R}^{P}\left(T^{\prime}\right)+1=\gamma_{R}\left(T^{\prime}\right)+1 .
$$

On the other hand, we can assume that $f(w)=2, f(u)=0$ and $f(v)=1$. Then $f^{\prime}$ is a RD-function on $T^{\prime}$, from which we deduce that $\gamma_{R}\left(T^{\prime}\right) \leq \gamma_{R}(T)-1$ and as above we have $\gamma_{R}(T)=\gamma_{R}^{P}(T)$.

Now we show that $\gamma_{R}(T) \equiv \gamma_{R}^{P}(T)$. Suppose, to the contrary, $\gamma_{R}(T) \not \equiv \gamma_{R}^{P}(T)$. Then there is a $\gamma_{R}(T)$-function $g$ such that $g$ is not a PRD-function. Without loss of generality, we assume that $g(w)=2, g(u)=0$ and $g(v)=1$. Hence $\left.g\right|_{T^{\prime}}$ is $\gamma_{R}\left(T^{\prime}\right)$-function that is not a PRD-function, contradicting the fact that $\gamma_{R}^{p}\left(T^{\prime}\right) \equiv$ $\gamma_{R}\left(T^{\prime}\right)$. Hence $\gamma_{R}(T) \equiv \gamma_{R}^{P}(T)$.

Case 9. $T$ is obtained from $T^{\prime}$ by Operation $\varphi_{9}$.

Let $r$ and $z$ are two weak support neighbors with degree two of $w$ and $N(r)=\{x, w\}$ and $N(z)=\{y, w\}$. Now assume that $g^{\prime}$ is a $\gamma_{R}^{P}\left(T^{\prime}\right)$-function. Since, $\gamma_{R}^{p}\left(T^{\prime}\right) \equiv \gamma_{R}\left(T^{\prime}\right)$, we can assume that $g^{\prime}(w)=2, g^{\prime}(u)=$ $g^{\prime}(r)=g^{\prime}(z)=0$ and $g^{\prime}(x)=g^{\prime}(y)=1$. Then $g^{\prime}$ can be extended to a PRD-function $g$ on $T$ by assigning to $v$ the value 1 , implying that $\gamma_{R}^{P}(T) \leq w(g)=w\left(g^{\prime}\right)+1=\gamma_{R}^{P}\left(T^{\prime}\right)+1$. Hence by the inductive hypothesis, we obtain

$$
\gamma_{R}(T) \leq \gamma_{R}^{P}(T) \leq w(g)=w\left(g^{\prime}\right)+1=\gamma_{R}^{P}\left(T^{\prime}\right)+1=\gamma_{R}\left(T^{\prime}\right)+1 .
$$

On the other hand, we can assume that $f(w)=2, f(u)=0$ and $f(v)=1$. Then $f^{\prime}$ is a RD-function on $T^{\prime}$, from which we deduce that $\gamma_{R}\left(T^{\prime}\right) \leq \gamma_{R}(T)-1$ and as above we have $\gamma_{R}(T)=\gamma_{R}^{P}(T)$.

Suppose, to the contrary, $\gamma_{R}(T) \not \equiv \gamma_{R}^{P}(T)$. Then there is a $\gamma_{R}(T)$-function $g$ such that $g$ is not a PRD-function. Without loss of generality, we assume that $g(w)=2, g(u)=0$ and $g(v)=1$. Hence $\left.g\right|_{T^{\prime}}$ is $\gamma_{R}\left(T^{\prime}\right)$-function that is not a PRD-function, contradicting the fact that $\gamma_{R}^{p}\left(T^{\prime}\right) \equiv \gamma_{R}\left(T^{\prime}\right)$. Hence $\gamma_{R}(T) \equiv \gamma_{R}^{P}(T)$.

Case 10. $T$ is obtained from $T^{\prime}$ by Operation $\varphi_{10}$.

Let $r$ be neighbor weak support vertex with degree two of $w$ and $N(r)=\{x, w\}$. Now assume that $g^{\prime}$ is a $\gamma_{R}^{P}\left(T^{\prime}\right)$-function. Since, $\gamma_{R}^{p}\left(T^{\prime}\right) \equiv \gamma_{R}\left(T^{\prime}\right)$, we can assume that $g^{\prime}(w)=2, g^{\prime}(u)=g^{\prime}(r)=0$ and $g^{\prime}(x)=1$. Then $g^{\prime}$ can be extended to a PRD-function $g$ on $T$ by assigning to $v$ the value 1 , implying that $\gamma_{R}^{P}(T) \leq w(g)=w\left(g^{\prime}\right)+1=\gamma_{R}^{P}\left(T^{\prime}\right)+1$. Hence by the inductive hypothesis, we obtain

$$
\gamma_{R}(T) \leq \gamma_{R}^{P}(T) \leq w(g)=w\left(g^{\prime}\right)+1=\gamma_{R}^{P}\left(T^{\prime}\right)+1=\gamma_{R}\left(T^{\prime}\right)+1 .
$$


On the other hand, we can assume that $f(w)=2, f(u)=0$ and $f(v)=1$. Then $f^{\prime}$ is a RD-function on $T^{\prime}$, from which we deduce that $\gamma_{R}\left(T^{\prime}\right) \leq \gamma_{R}(T)-1$. Consequently, we must have equalities throughout the inequality chain (3.10). In particular, $\gamma_{R}(T)=\gamma_{R}^{P}(T)$.

Suppose, to the contrary, $\gamma_{R}(T) \not \equiv \gamma_{R}^{P}(T)$. Then there is a $\gamma_{R}(T)$-function $g$ such that $g$ is not a PRDfunction. Without loss of generality, we assume that $g(w)=2, g(u)=0$ and $g(v)=1$. Hence $\left.g\right|_{T^{\prime}}$ is $\gamma_{R}\left(T^{\prime}\right)$-function that is not a PRD-function, contradicting thr fact $\gamma_{R}^{p}\left(T^{\prime}\right) \equiv \gamma_{R}\left(T^{\prime}\right)$. Hence $\gamma_{R}(T) \equiv \gamma_{R}^{P}(T)$.

Case 11. $T$ is obtained from $T^{\prime}$ by Operation $\varphi_{11}$.

Let $g^{\prime}$ be a $\gamma_{R}^{P}\left(T^{\prime}\right)$-function. Then $g^{\prime}$ can be extended to a PRD-function $g$ on $T$ by assigning to $v$ and $y$ the value 0 and to $x$ the value 2 . Hence $\gamma_{R}^{P}(T) \leq w(g)=w\left(g^{\prime}\right)+2=\gamma_{R}^{P}\left(T^{\prime}\right)+2$. Hence by the inductive hypothesis, we obtain

$$
\gamma_{R}(T) \leq \gamma_{R}^{P}(T) \leq w(g)=w\left(g^{\prime}\right)+2=\gamma_{R}^{P}\left(T^{\prime}\right)+2=\gamma_{R}\left(T^{\prime}\right)+2 .
$$

On the other hand, we can assume that $f(x)=2$ and $f(v)=0$. Then $f^{\prime}$ is a RD-function on $T^{\prime}$, from which we deduce that $\gamma_{R}\left(T^{\prime}\right) \leq \gamma_{R}(T)-1$. Consequently, we must have equalities throughout the inequality chain (3.11). In particular, $\gamma_{R}(T)=\gamma_{R}^{P}(T)$.

Suppose, to the contrary, $\gamma_{R}(T) \not \equiv \gamma_{R}^{P}(T)$. Then there is a $\gamma_{R}(T)$-function $g$ such that $g$ is not a PRD-function. Without loss of generality, we assume that $g(x)=2$ and $g(v)=0$. Hence $\left.g\right|_{T^{\prime}}$ is $\gamma_{R}\left(T^{\prime}\right)$-function and so $g(u) \neq 2$. Therefore $\left.g\right|_{T^{\prime}}$ is not a PRD-function, contradicting the fact that $\gamma_{R}^{p}\left(T^{\prime}\right) \equiv \gamma_{R}\left(T^{\prime}\right)$. Hence $\gamma_{R}(T) \equiv \gamma_{R}^{P}(T)$ and the proof is complete.

Now we are ready to establish our main result.

Theorem 3.4. Let $T$ be a tree. Then $\gamma_{R}(T) \equiv \gamma_{R}^{P}(T)$ if and only if $T$ is $K_{1}$ or $T \in \mathcal{F}$.

Proof. The sufficiency follows from Lemma 3.3. To prove the necessity, we proceed by induction on the order $n$ of a tree $T$ that satisfying $\gamma_{R}(T) \equiv \gamma_{R}^{P}(T)$. Clearly if $n=1$, then $T=K_{1}$. Hence we assume that $T$ has order $n \geq 2$. If $n \leq 3$, then $T \in\left\{P_{2}, P_{3}\right\}$ and clearly $\gamma_{R}(T) \equiv \gamma_{R}^{P}(T)$ and $T \in \mathcal{F}$. Thus, we assume that $n \geq 4$. Assume that every tree $T^{\prime}$ of order $2 \leq n^{\prime}<n$ with $\gamma_{R}\left(T^{\prime}\right) \equiv \gamma_{R}^{P}\left(T^{\prime}\right)$ is in $\mathcal{F}$. Let $T$ be a tree of order $n$ with $\gamma_{R}(T) \equiv \gamma_{R}^{P}(T)$ and let $f$ be a $\gamma_{R}(T)$-function. If $T$ is a star, then $T$ can be obtained from $P_{3}$ by repeated applications of operation $\varphi_{1}$ noting that the central vertex of a star on at least three vertices is a strong support vertex of the star, implying that $T \in \mathcal{F}$. Hence, we may assume that $\operatorname{diam}(T) \geq 3$. We proceed further with the following claims.

Claim 3.5. If $\operatorname{diam}(T)=3$, then $T \in \mathcal{F}$.

Proof of Claim 3.5. Suppose that $\operatorname{diam}(T)=3$. Then $T$ is a double star $S(r, s)$ for some integers $r \geq s \geq 1$. Let $u$ and $v$ be the non-leaf vertices of $T$ such that $\operatorname{deg}(v)=s+1$ and $\operatorname{deg}(u)=r+1$. Suppose first that $s=1$ and let $w$ be the leaf-neighbor of $v$. Thus, $T$ can be obtained from a path $P_{3}$ with central vertex $v$ by first applying operation $\varphi_{7}$ with $u$ as the attacher, thereby producing a double star $S(2,1)$, and then by repeated applications of operation $\varphi_{1}$, implying that $T \in \mathcal{F}$. Now assume that $s \geq 2$. Then, $T$ can be obtained from a path $P_{3}$ with central vertex $u$ by first applying operation $\varphi_{6}$ with $u$ as the attacher, thereby producing a double star $S(2,1)$, and then by repeated applications of operation $\varphi_{1}$, implying that $T \in \mathcal{F}$.

By Claim 3.5, we may assume that $\operatorname{diam}(T) \geq 4$.

Claim 3.6. If $T$ contains a support vertex with at least three leaf neighbors, then $T \in \mathcal{F}$.

Proof of Claim 3.6. Suppose that $T$ contains a support vertex $u$ with at least three leaf neighbors. Let $v$ be an arbitrary leaf neighbor of $u$, and let $T^{\prime}=T-v$. Since $u$ has at least two leaf neighbors in $T^{\prime}$, the vertex $u$ is a strong support vertex in $T^{\prime}$. Clearly $f(u)=2$ and $f(v)=0$. Hence $\left.f\right|_{T^{\prime}}$ is a PRD-function for tree $T^{\prime}$ and so

$$
\gamma_{R}\left(T^{\prime}\right) \leq \gamma_{R}^{P}\left(T^{\prime}\right) \leq \gamma_{R}^{P}(T)=\gamma_{R}(T)
$$


Now assume that $g^{\prime}$ is a $\gamma_{R}\left(T^{\prime}\right)$-function, then we can assume that $g^{\prime}(u)=2$. Then $g^{\prime}$ can be extended to a RD-function on $T$ by assigning the weight 0 to the vertex $v$, implying that $\gamma_{R}(T) \leq \gamma_{R}\left(T^{\prime}\right)$. Consequently, $\gamma_{R}(T) \leq \gamma_{R}\left(T^{\prime}\right)$ and so we must have equalities throughout the inequality chain (3.12). In particular, $\gamma_{R}\left(T^{\prime}\right)=$ $\gamma_{R}^{P}\left(T^{\prime}\right)$. On the other hand, if $\gamma_{R}\left(T^{\prime}\right) \not \equiv \gamma_{R}^{P}\left(T^{\prime}\right)$, then there exists $\gamma_{R}\left(T^{\prime}\right)$-function $g^{\prime}$ such that $g^{\prime}$ is not a PRD-function. Since $u$ is a strong support vertex, we can assume that $g^{\prime}(u)=2$. Then $g^{\prime}$ can be extended to a $\gamma_{R}(T)$-function $g$ by assigning 0 to $v$. Then $g$ is not a PRD-function, contradiction to our assumption that $\gamma_{R}(T) \equiv \gamma_{R}^{P}(T)$. Therefore $\gamma_{R}\left(T^{\prime}\right) \equiv \gamma_{R}^{P}\left(T^{\prime}\right)$ and by induction on $T^{\prime}$, we have $T^{\prime} \in \mathcal{F}$. Since the vertex $u$ is a strong support vertex in $T^{\prime}$, the tree $T$ can be rebuilt from the tree $T^{\prime}$ by applying Operation $\varphi_{1}$ with $u$ as the attacher. Thus, $T \in \mathcal{F}$.

By Claim 3.6, we may assume that every support vertex in $T$ has at most two leaf neighbors. We now root the tree $T$ at a vertex $r$ at the end of a longest path in $T$. Let $v$ be a vertex at maximum distance from $r$, and so $d_{T}(v, r)=\operatorname{diam}(T)$. Necessarily, $r$ and $v$ are leaves. Let $u$ be the parent of $v$, let $w$ be the parent of $u$, let $x$ be the parent of $w$, and let $y$ be the parent of $x$. Possibly, $y=r$. Since $v$ is a vertex at maximum distance from the root $r$, every child of $u$ is a leaf. Thus by our earlier observations, $d_{T}(u) \leq 3$. Among all $\gamma_{R}(T)$-functions, let $f$ be chosen so that the sum of the values assigned to all leaves under $f$ is minimum. Since $\gamma_{R}(T) \equiv \gamma_{R}^{P}(T)$, it follow that $f$ is a $\gamma_{R}^{P}(T)$-functions. Throughout the remaining part of the proof, if $T^{\prime}$ is a subtree of $T$, then we let $f^{\prime}$ be the restriction of the function $f$ to the tree $T^{\prime}$. Thus, $f^{\prime}(z)=f(z)$ for every vertex $z \in V\left(T^{\prime}\right)$.

Claim 3.7. If $\operatorname{deg}(u)=3$, then $T \in \mathcal{F}$.

Proof of Claim 3.7. Then clearly we can see that $f(u)=2$ and so by Proposition $3.1, f(w)=2$ or $f(w)=0$. We consider two cases.

Case 3.1. $f(w)=2$.

It follows that $\operatorname{deg}(w) \geq 3$. Then every child of $w$ is a leaf or a support vertex. We first assume that $w$ is not a weak support vertex. Let $T^{\prime}=T-T_{u}$. Since $\gamma_{R}(T) \equiv \gamma_{R}^{P}(T)$, we deduce that $f$ is a $\gamma_{R}^{P}(T)$-function. Then $\left.f\right|_{T^{\prime}}$ is a PRD-function for tree $T^{\prime}$ and so

$$
\gamma_{R}\left(T^{\prime}\right) \leq \gamma_{R}^{P}\left(T^{\prime}\right) \leq \gamma_{R}^{P}(T)-2=\gamma_{R}(T)-2 .
$$

Now assume that $g^{\prime}$ is a $\gamma_{R}\left(T^{\prime}\right)$-function. Then $g^{\prime}$ can be extended to a RD-function on $T$ by assigning the weight 2 to $u$ and 0 to the remaining vertices in $V\left(T_{u}\right)$, implying that $\gamma_{R}(T) \leq \gamma_{R}\left(T^{\prime}\right)+2$. Consequently, $\gamma_{R}(T)=\gamma_{R}\left(T^{\prime}\right)+2$ and so we must have equalities throughout the inequality chain (3.13). In particular, $\gamma_{R}\left(T^{\prime}\right)=\gamma_{R}^{P}\left(T^{\prime}\right)$. On the other hand, if $\gamma_{R}\left(T^{\prime}\right) \not \equiv \gamma_{R}^{P}\left(T^{\prime}\right)$, then there exists $\gamma_{R}\left(T^{\prime}\right)$-function $g^{\prime}$ such that $g^{\prime}$ is not a PRD-function. Then $g^{\prime}$ can be extended to a $\gamma_{R}(T)$-function by assigning 2 to $u$ and 0 to the remaining vertices in $V\left(T_{u}\right)$, that is not a PRD-function on tree $T$, contradicting the fact that $\gamma_{R}(T) \equiv \gamma_{R}^{P}(T)$. Therefore $\gamma_{R}\left(T^{\prime}\right) \equiv \gamma_{R}^{P}\left(T^{\prime}\right)$ and by applying the inductive hypothesis to $T^{\prime}$, we have $T^{\prime} \in \mathcal{F}$.

Now assume that $g^{\prime}$ is a $\gamma_{R}\left(T^{\prime}\right)$-function. If $g^{\prime}(w)=1$, then $g^{\prime}$ can be extended to a RD-function of $T$ by reassigning to $w$ the weight 0 , assigning to $u$ the weight 2 and to the remaining vertices in $V\left(T_{u}\right)$ the weight 0 . Then $\gamma_{R}(T) \leq w\left(g^{\prime}\right)+1=\gamma_{R}\left(T^{\prime}\right)+1=\gamma_{R}(T)-1$, a contradiction. Now assume that $g^{\prime}(w)=0$, then $g^{\prime}$ can be extended to a $\gamma_{R}(T)$-function by assigning 2 to $u$ and 0 to the remaining vertices in $V\left(T_{u}\right)$, that is not a PRD-function on tree $T$, contradicting the fact that $\gamma_{R}(T) \equiv \gamma_{R}^{P}(T)$. Hence, every $\gamma_{R}\left(T^{\prime}\right)$-function assigns 2 to the vertex $w$. Further in this case, if $w$ has at least one child different from $u$ that is a strong support vertex, then the tree $T$ can be rebuilt from the tree $T^{\prime}$ by applying Operation $\mathcal{O}_{4}$ with $w$ as the attacher. Thus, $T \in \mathcal{F}$. Hence, we may assume that every child of $w$ is a leaf or a weak support vertex. If $w$ has at least one child that is a weak support vertex, then the tree $T$ can be rebuilt from the tree $T^{\prime}$ by applying Operation $\mathcal{O}_{5}$ with $w$ as the attacher. Thus, $T \in \mathcal{F}$. Hence, we may assume that $w$ is a support vertex. If $v$ is a strong support vertex, then the tree $T$ can be rebuilt from the tree $T^{\prime}$ by applying Operation $\mathcal{O}_{6}$ with $w$ as the attacher and so $T \in \mathcal{F}$. Now assume that $w$ is a weak support vertex. Let $T^{\prime}=T-T_{w}$ and 
$L(w)=\{z\}$. In this case, reassigning to $w$ the weight 0 , to $z, x$ the weight 1 and leaving all other weights unchanged produces a new $\gamma_{R}^{P}(T)$-function $h$ such that $\left.h\right|_{T^{\prime}}$ is a PRD-function for tree $T^{\prime}$. Hence,

$$
\gamma_{R}\left(T^{\prime}\right) \leq \gamma_{R}^{P}\left(T^{\prime}\right) \leq \gamma_{R}^{P}(T)-3=\gamma_{R}(T)-3 .
$$

Now assume that $g^{\prime}$ is a $\gamma_{R}\left(T^{\prime}\right)$-function. Then $g^{\prime}$ can be extended to a RD-function on $T$ by assigning the weight 2 to $u$, the weight 1 to $z$ and to the remaining vertices in $V\left(T_{w}\right)$ the weight 0 , implying that $\gamma_{R}(T) \leq \gamma_{R}\left(T^{\prime}\right)+3$. Consequently, $\gamma_{R}(T)=\gamma_{R}\left(T^{\prime}\right)+3$ and so we must have equalities throughout the inequality chain (3.14). In particular, $\gamma_{R}\left(T^{\prime}\right)=\gamma_{R}^{P}\left(T^{\prime}\right)$.

On the other hand, if $\gamma_{R}\left(T^{\prime}\right) \not \equiv \gamma_{R}^{P}\left(T^{\prime}\right)$, then there exists $\gamma_{R}\left(T^{\prime}\right)$-function $g^{\prime}$ such that $g^{\prime}$ is not a PRDfunction. Then $g^{\prime}$ can be extended to a $\gamma_{R}(T)$-function by assigning to $u$ the weight 2 , the weight 1 to $z$ and to the remaining vertices in $V\left(T_{w}\right)$ the weight 0 , contradicting the fact that $\gamma_{R}(T) \equiv \gamma_{R}^{P}(T)$. Therefore $\gamma_{R}\left(T^{\prime}\right) \equiv \gamma_{R}^{P}\left(T^{\prime}\right)$ and so by applying the inductive hypothesis to $T^{\prime}$, we have $T^{\prime} \in \mathcal{F}$.

If there exists a $\gamma_{R}\left(T^{\prime}\right)$-function $g^{\prime}$ such that $g^{\prime}(x)=2$, such a function $g^{\prime}$ can be extended to a RD-function of $T$ by assigning to $u$ the weight 2 , the weight 1 to $z$ and to the remaining vertices in $V\left(T_{w}\right)$ the weight 0 , that is not a PRD-function on tree $T$, contradicting the fact that $\gamma_{R}(T) \equiv \gamma_{R}^{P}(T)$. Hence, $x$ cannot be assigned the value 2 under any $\gamma_{R}\left(T^{\prime}\right)$-function. Hence the tree $T$ can be rebuilt from the tree $T^{\prime}$ by applying Operation $\mathcal{O}_{3}$ with $x$ as the attacher.

Case 3.2. $f(w)=0$.

We know that $f$ is a $\gamma_{R}^{P}(T)$-function and so for every neighbors $z$ of $w$ different from $u, f(z) \neq 2$. Hence no child of $w$ different from $u$ is a support vertex. If $w$ is a strong support vertex. Then reassigning to $w$ the weight 2 and to leaves neighbors of it the weight 0 , and leaving all other weights unchanged produces a new $\gamma_{R}(T)$-function such that the sum of the values assigned to all leaves is smaller than the sum under $f$, a contradiction. Hence in this case, $w$ is a weak support vertex or $\operatorname{deg}(w)=2$. We first assume that $w$ is a weak support vertex. Let $L(w)=\{z\}$. Clearly, $f(z)=1$. Let $T^{\prime}=T-T_{w}$. Then $\left.f\right|_{T^{\prime}}$ is a PRD-function for tree $T^{\prime}$ and so

$$
\gamma_{R}\left(T^{\prime}\right) \leq \gamma_{R}^{P}\left(T^{\prime}\right) \leq \gamma_{R}^{P}(T)-3=\gamma_{R}(T)-3 .
$$

Now assume that $g^{\prime}$ is a $\gamma_{R}\left(T^{\prime}\right)$-function. Then $g^{\prime}$ can be extended to a RD-function on $T$ by assigning the weight 2 to $u$, to $z$ the weight 1 and to the remaining vertices in $V\left(T_{w}\right)$ the weight 0 , implying that $\gamma_{R}(T) \leq \gamma_{R}\left(T^{\prime}\right)+3$. Consequently, $\gamma_{R}\left(T^{\prime}\right)=\gamma_{R}^{P}\left(T^{\prime}\right)$.

On the other hand, if $\gamma_{R}\left(T^{\prime}\right) \not \equiv \gamma_{R}^{P}\left(T^{\prime}\right)$, then there exists $\gamma_{R}\left(T^{\prime}\right)$-function $g^{\prime}$ such that $g^{\prime}$ is not a PRDfunction. Then $g^{\prime}$ can be extended to a $\gamma_{R}(T)$-function by assigning to $u$ the weight 2 , to $z$ the weight 1 , and to the remaining vertices in $V\left(T_{w}\right)$ the weight 0 , that is not a PRD-function on tree $T$, contradicting the fact that $\gamma_{R}(T) \equiv \gamma_{R}^{P}(T)$. Therefore $\gamma_{R}\left(T^{\prime}\right) \equiv \gamma_{R}^{P}\left(T^{\prime}\right)$ and by the inductive hypothesis to $T^{\prime}$, we have $T^{\prime} \in \mathcal{F}$.

If there exists a $\gamma_{R}\left(T^{\prime}\right)$-function $f^{\prime}$ such that $f^{\prime}(x)=2$, then such a function $f^{\prime}$ can be extended to a $\gamma_{R}(T)$-function of $T$ by assigning 2 to $u, 1$ to $z$ and 0 to the remaining vertices in $V\left(T_{w}\right)$, that is not a PRD-function on tree $T$, contradicting the fact that $\gamma_{R}(T) \equiv \gamma_{R}^{P}(T)$. Hence, no $\gamma_{R}\left(T^{\prime}\right)$-function assigns to the vertex $x$ the weight 2 . The tree $T$ can be rebuilt from the tree $T^{\prime}$ by applying Operation $\mathcal{O}_{3}$ with $x$ as the attacher. Thus, $T \in \mathcal{F}$.

Next assume that $\operatorname{deg}(w)=2$. Let $T^{\prime}=T-T_{w}$. Then $\left.f\right|_{T^{\prime}}$ is a PRD-function for tree $T^{\prime}$ and so

$$
\gamma_{R}\left(T^{\prime}\right) \leq \gamma_{R}^{P}\left(T^{\prime}\right) \leq \gamma_{R}^{P}(T)-2=\gamma_{R}(T)-2 .
$$

On the other hand, any $\gamma_{R}\left(T^{\prime}\right)$-function can be extended to a RD-function on $T$ by assigning a 2 to $u$ and 0 to the remaining vertices in $V\left(T_{w}\right)$, implying that $\gamma_{R}(T) \leq \gamma_{R}\left(T^{\prime}\right)+2$ implying that $\gamma_{R}(T)=\gamma_{R}\left(T^{\prime}\right)+2$ and as above we obtain $\gamma_{R}\left(T^{\prime}\right)=\gamma_{R}^{P}\left(T^{\prime}\right)$.

If $\gamma_{R}\left(T^{\prime}\right) \not \equiv \gamma_{R}^{P}\left(T^{\prime}\right)$, then any $\gamma_{R}\left(T^{\prime}\right)$-function which is not a PRD-function, can be extended to a $\gamma_{R}(T)$ function by assigning the weight 2 to $u$ and the weight 0 to the remaining vertices of $T_{w}$, that is not a PRD-function on tree $T$, contradicting the fact that $\gamma_{R}(T) \equiv \gamma_{R}^{P}(T)$. Therefore $\gamma_{R}\left(T^{\prime}\right) \equiv \gamma_{R}^{P}\left(T^{\prime}\right)$ and by 
the inductive hypothesis to $T^{\prime}$, we have $T^{\prime} \in \mathcal{F}$. Now the tree $T$ can be rebuilt from the tree $T^{\prime}$ by applying Operation $\mathcal{O}_{2}$ with $x$ as the attacher and so $T \in \mathcal{F}$.

Claim 3.8. If $\operatorname{deg}(u)=2$, then $T \in \mathcal{F}$.

Proof of Claim 3.8. Then, every child of $w$ is a leaf or a support vertex. We distinguish two situations.

Case $4.1 w$ is a support vertex.

We first assume that $w$ is a strong support vertex. Let $T^{\prime}=T-\{u, v\}$. Without loss of generality, we can assume that $f(w)=2, f(u)=0$ and $f(v)=1$. Then $\left.f\right|_{T^{\prime}}$ is a PRD-function and so

$$
\gamma_{R}\left(T^{\prime}\right) \leq \gamma_{R}^{P}\left(T^{\prime}\right) \leq \gamma_{R}^{P}(T)-1=\gamma_{R}(T)-1 .
$$

Now assume that $g^{\prime}$ is a $\gamma_{R}\left(T^{\prime}\right)$-function, then we may assume that $g^{\prime}(w)=2$, since $w$ is a strong support vertex. Then $g^{\prime}$ can be extended to a RD-function on $T$ by assigning the weight 0 to $u$ and 1 to $v$, implying that $\gamma_{R}(T) \leq \gamma_{R}\left(T^{\prime}\right)+1$. Consequently, $\gamma_{R}(T)=\gamma_{R}\left(T^{\prime}\right)+1$ and by $(3.17)$ we have $\gamma_{R}\left(T^{\prime}\right)=\gamma_{R}^{P}\left(T^{\prime}\right)$.

On the other hand, if $\gamma_{R}\left(T^{\prime}\right) \not \equiv \gamma_{R}^{P}\left(T^{\prime}\right)$, then there exists $\gamma_{R}\left(T^{\prime}\right)$-function $g^{\prime}$ such that $g^{\prime}$ is not a PRDfunction. We can assume that $g^{\prime}(w)=2$, since $w$ is a strong support vertex. Then $g^{\prime}$ can be extended to a $\gamma_{R}(T)$-function by assigning the weight 0 to $u$ and 1 to $v$, that is not a PRD-function on tree $T$, contradicting the fact that $\gamma_{R}(T) \equiv \gamma_{R}^{P}(T)$. Therefore $\gamma_{R}\left(T^{\prime}\right) \equiv \gamma_{R}^{P}\left(T^{\prime}\right)$ and by the inductive hypothesis on $T^{\prime}$, we have $T^{\prime} \in \mathcal{F}$. The tree $T$ can be rebuilt from the tree $T^{\prime}$ by applying Operation $\mathcal{O}_{7}$ with $w$ as the attacher. Thus, $T \in \mathcal{F}$.

Now assume that $w$ is a weak support vertex. Let $T^{\prime}=T-v$. It is easy to see that

$$
\gamma_{R}\left(T^{\prime}\right) \leq \gamma_{R}^{P}\left(T^{\prime}\right) \leq \gamma_{R}^{P}(T)-1=\gamma_{R}(T)-1 .
$$

Now assume that $g^{\prime}$ is a $\gamma_{R}\left(T^{\prime}\right)$-function, then we may assume that $g^{\prime}(w)=2$, since $w$ is a strong support vertex. Then $g^{\prime}$ can be extended to a RD-function on $T$ by assigning the weight 1 to $v$, implying that $\gamma_{R}(T) \leq \gamma_{R}\left(T^{\prime}\right)+1$. Consequently, $\gamma_{R}(T)=\gamma_{R}\left(T^{\prime}\right)+1$ and so we must have equalities throughout the inequality chain (3.18). In particular, $\gamma_{R}\left(T^{\prime}\right)=\gamma_{R}^{P}\left(T^{\prime}\right)$.

On the other hand, if $\gamma_{R}\left(T^{\prime}\right) \not \equiv \gamma_{R}^{P}\left(T^{\prime}\right)$, then there exists $\gamma_{R}\left(T^{\prime}\right)$-function $g^{\prime}$ such that $g^{\prime}$ is not a PRDfunction. Then $g^{\prime}$ can be extended to a $\gamma_{R}(T)$-function by assigning the weight 1 to $v$, that is not a PRDfunction on tree $T$, contradicting the fact that $\gamma_{R}(T) \equiv \gamma_{R}^{P}(T)$. Therefore $\gamma_{R}\left(T^{\prime}\right) \equiv \gamma_{R}^{P}\left(T^{\prime}\right)$ and so by applying the inductive hypothesis on $T^{\prime}$, we have $T^{\prime} \in \mathcal{F}$. Now $T$ can be rebuilt from the tree $T^{\prime}$ by applying Operation $\mathcal{O}_{8}$ with $u$ as the attacher and so $T \in \mathcal{F}$.

Case 4.2. $w$ is not a support vertex.

Then every child of $w$ is a weak support vertex. We first assume that $\operatorname{deg}(w) \geq 4$. Let $T^{\prime}=T-v$. Without loss of generality, we can assume that $f(w)=2$, and every support vertex adjacent to $w$ has weight 0 and their leaf neighbors have weight 1 . Then $\left.f\right|_{T^{\prime}}$ is a PRD-function and so

$$
\gamma_{R}\left(T^{\prime}\right) \leq \gamma_{R}^{P}\left(T^{\prime}\right) \leq \gamma_{R}^{P}(T)-1=\gamma_{R}(T)-1 .
$$

Now assume that $g^{\prime}$ is a $\gamma_{R}\left(T^{\prime}\right)$-function. Then $g^{\prime}$ can be extended to a RD-function on $T$ by assigning the weight 1 to $v$, implying that $\gamma_{R}(T) \leq \gamma_{R}\left(T^{\prime}\right)+1$. Consequently, $\gamma_{R}(T)=\gamma_{R}\left(T^{\prime}\right)+1$ and using (3.19) we obtain $\gamma_{R}\left(T^{\prime}\right)=\gamma_{R}^{P}\left(T^{\prime}\right)$.

On the other hand, if $\gamma_{R}\left(T^{\prime}\right) \not \equiv \gamma_{R}^{P}\left(T^{\prime}\right)$, then there exists $\gamma_{R}\left(T^{\prime}\right)$-function $g^{\prime}$ such that $g^{\prime}$ is not a PRDfunction. Then $g^{\prime}$ can be extended to a $\gamma_{R}(T)$-function by assigning the weight 1 to $v$, that is not a PRDfunction on tree $T$, contradicting the fact that $\gamma_{R}(T) \equiv \gamma_{R}^{P}(T)$. Therefore $\gamma_{R}\left(T^{\prime}\right) \equiv \gamma_{R}^{P}\left(T^{\prime}\right)$ and so $T^{\prime} \in \mathcal{F}$ by the inductive hypothesis on $T^{\prime}$. The tree $T$ can be rebuilt from the tree $T^{\prime}$ by applying Operation $\mathcal{O}_{9}$ with $u$ as the attacher. Thus, $T \in \mathcal{F}$. 
Now assume that $\operatorname{deg}(w)=3$. Let $T^{\prime}=T-v$. Without loss of generality, we can assume that $f(w)=2$, and every support vertex adjacent to $w$ has weight 0 and their leaf neighbors have weight 1 . Then $\left.f\right|_{T^{\prime}}$ is a PRD-function and so

$$
\gamma_{R}\left(T^{\prime}\right) \leq \gamma_{R}^{P}\left(T^{\prime}\right) \leq \gamma_{R}^{P}(T)-1=\gamma_{R}(T)-1 .
$$

Now assume that $g^{\prime}$ is a $\gamma_{R}\left(T^{\prime}\right)$-function. Then $g^{\prime}$ can be extended to a RD-function on $T$ by assigning the weight 1 to $v$, implying that $\gamma_{R}(T) \leq \gamma_{R}\left(T^{\prime}\right)+1$. Consequently, $\gamma_{R}(T)=\gamma_{R}\left(T^{\prime}\right)+1$ and so we must have equalities throughout the inequality chain (3.20). In particular, $\gamma_{R}\left(T^{\prime}\right)=\gamma_{R}^{P}\left(T^{\prime}\right)$.

On the other hand, if $\gamma_{R}\left(T^{\prime}\right) \not \equiv \gamma_{R}^{P}\left(T^{\prime}\right)$, then there exists $\gamma_{R}\left(T^{\prime}\right)$-function $g^{\prime}$ such that $g^{\prime}$ is not a PRDfunction. Then $g^{\prime}$ can be extended to a $\gamma_{R}(T)$-function by assigning the weight 1 to $v$, that is not a PRDfunction on tree $T$, contradicting the fact that $\gamma_{R}(T) \equiv \gamma_{R}^{P}(T)$. Therefore $\gamma_{R}\left(T^{\prime}\right) \equiv \gamma_{R}^{P}\left(T^{\prime}\right)$ and so by applying the inductive hypothesis to $T^{\prime}$, the tree $T^{\prime} \in \mathcal{F}$. The tree $T$ can be rebuilt from the tree $T^{\prime}$ by applying Operation $\mathcal{O}_{9}$ with $u$ as the attacher. Thus, $T \in \mathcal{F}$.

If there exists a $\gamma_{R}\left(T^{\prime}\right)$-function $f^{\prime}$ such that $f^{\prime}(w) \neq 2$, then it is clear that $f^{\prime}(w)=0$ and $f^{\prime}(u)=1$. Such a function $f^{\prime}$ can be extended to a RD-function of $T$ by assigning to $v$ the weight 0 and reassigning to $u$ the weight 2 , that is not a PRD-function on tree $T$, contradicting the fact that $\gamma_{R}(T) \equiv \gamma_{R}^{P}(T)$. Hence, every $\gamma_{R}\left(T^{\prime}\right)$-function assigns to the vertex $w$ the weight 2 . The tree $T$ can be rebuilt from the tree $T^{\prime}$ by applying Operation $\mathcal{O}_{10}$ with $u$ as the attacher. Thus, $T \in \mathcal{F}$.

Next assume that $\operatorname{deg}(w)=2$. Let $T^{\prime}=T-\{v, u, w\}$. Without loss of generality, we can assume that $f(w)=f(v)=0$ and $f(u)=2$. Then $\left.f\right|_{T^{\prime}}$ is a PRD-function and so

$$
\gamma_{R}\left(T^{\prime}\right) \equiv \gamma_{R}^{P}\left(T^{\prime}\right) \leq \gamma_{R}^{P}(T)-2=\gamma_{R}(T)-2 .
$$

Now assume that $g^{\prime}$ is a $\gamma_{R}\left(T^{\prime}\right)$-function. Then $g^{\prime}$ can be extended to a RD-function on $T$ by assigning the weight 2 to $u$ and to $v$ and $w$ the weight 0 , implying that $\gamma_{R}(T) \leq \gamma_{R}\left(T^{\prime}\right)+2$. Consequently, $\gamma_{R}(T)=\gamma_{R}\left(T^{\prime}\right)+$ 2 and so we must have equalities throughout the inequality chain (3.21). In particular, $\gamma_{R}\left(T^{\prime}\right)=\gamma_{R}^{P}\left(T^{\prime}\right)$.

On the other hand, if $\gamma_{R}\left(T^{\prime}\right) \not \equiv \gamma_{R}^{P}\left(T^{\prime}\right)$, then there exists $\gamma_{R}\left(T^{\prime}\right)$-function $g^{\prime}$ such that $g^{\prime}$ is not a PRD-function. Then $g^{\prime}$ can be extended to a $\gamma_{R}(T)$-function by assigning the weight 2 to $u$ and to $v$ and $w$ the weight 0 , that is not a PRD-function on tree $T$, contradicting the fact that $\gamma_{R}(T) \equiv \gamma_{R}^{P}(T)$. Therefore $\gamma_{R}\left(T^{\prime}\right) \equiv \gamma_{R}^{P}\left(T^{\prime}\right)$ and so by applying the inductive hypothesis to $T^{\prime}$, the tree $T^{\prime} \in \mathcal{F}$. If there exists a $\gamma_{R}\left(T^{\prime}\right)$-function $f^{\prime}$ such that $f^{\prime}(x)=2$, then function $f^{\prime}$ can be extended to a RD-function of $T$ by assigning the weight 2 to $u$ and to $v$ and $w$ the weight 0 , that is not a PRD-function on tree $T$, contradicting the fact that $\gamma_{R}(T) \equiv \gamma_{R}^{P}(T)$. Hence, $x$ cannot be assigned the value 2 under any $\gamma_{R}\left(T^{\prime}\right)$-function. Then tree $T$ can be rebuilt from the tree $T^{\prime}$ by applying Operation $\mathcal{O}_{11}$ with $x$ as the attacher. Thus, $T \in \mathcal{F}$. This completes the proof of theorem.

Acknowledgements. This work was supported by the Natural Science Foundation of China under grant 62172116 and the Natural Science Foundation of Guangdong Province under Grant 2021A1515011940.

\section{REFERENCES}

[1] J. Amjadi, M. Falahat, M. Chellali and S.M. Sheikholeslami, Unicyclic graphs with strong equality between the 2-rainbow domination and independent 2-rainbow domination numbers. Trans. Comb. 4 (2015) 1-11.

[2] J. Amjadi, M. Falahat, S.M. Sheikholeslami and N. Jafari Rad, Strong equality between the 2-rainbow domination and independent 2-rainbow domination numbers in trees. Bull. Malays. Math. Sci. Soc. 39 (2016) 205-218.

[3] J.D. Alvarado, S. Dantas and D. Rautenbach, Strong equality of Roman and weak Roman domination in trees. Discrete Appl. Math. 208 (2016) 19-26.

[4] A. Cabrera Martínez, A. Martínez Arias and M. Menendez Castillo, A characterization relating domination, semitotal domination and total Roman domination in trees. Commun. Comb. Optim. 6 (2021) 197-209.

[5] M. Chellali, N. Jafari Rad, S.M. Sheikholeslami and L. Volkmann, Roman domination in graphs. In: Topics in Domination in Graphs, edited by T.W. Haynes, S.T. Hedetniemi and M.A. Henning. Springer, Berlin/Heidelberg (2020) 365-409.

[6] M. Chellali, N. Jafari Rad, S.M. Sheikholeslami and L. Volkmann, Varieties of Roman domination II. AKCE Int. J. Graphs Comb. 17 (2020) 966-984. 
[7] M. Chellai, N. Jafari Rad, S.M. Sheikholeslami, L. Volkmann, A survey on Roman domination parameters in directed graphs. J. Combin. Math. Combin. Comput. 115 (2020) 141-171.

[8] M. Chellali, N. Jafari Rad, S.M. Sheikholeslami and L. Volkmann, Varieties of Roman domination. In: Structures of Domination in Graphs, edited by T.W. Haynes, S.T. Hedetniemi and M.A. Henning. Springer, Berlin/Heidelberg (2021) $273-307$.

[9] M. Chellai, N. Jafari Rad, S.M. Sheikholeslami and L. Volkmann, The Roman domatic problem in graphs and digraphs: a survey. Discuss. Math. Graph Theory (to appear).

[10] M. Chellali, S.M. Sheikholeslami and M. Soroudi, A characterization of perfect Roman trees. Discrete Appl. Math. 285 (2020) $501-508$.

[11] E.J. Cockayne, P.M. Dreyer Jr., S.M. Hedetniemi and S.T. Hedetniemi, On Roman domination in graphs. Discrete Math. 278 (2004) 11-22.

[12] M.R. Garey and D.S. Johnson, Computers and Intractability: A Guide to the Theory of NP-Completeness. Freeman, San Francisco (1979).

[13] T.W. Haynes, M.A. Henning and P.J. Slater, Strong equality of domination parameters in trees. Discrete Math. 260 (2003) $77-87$.

[14] M.A. Henning and S.T. Hedetniemi, Defending the Roman empire - new strategy. Discrete Math. 266 (2003) $239-251$.

[15] M.A. Henning, W.F. Klostermeyer and G. MacGillivray, Perfect Roman domination in trees. Discrete Appl. Math. 236 (2018) 235-245.

[16] N. Jafari Rad and C.-H. Liu, Trees with strong equality between the Roman domination number and the unique response Roman domination number. Australas. J. Combin. 54 (2012) 133-140.

[17] S. Nazari-Moghaddam and S.M. Sheikholeslami, On trees with equal Roman domination and outer-independent Roman domination number. Commun. Comb. Optim. 4 (2019) 185-199.

[18] Z. Shao, S. Kosari, M. Chellali, S.M. Sheikholeslami and M. Soroudi, On a relation between the perfect Roman domination and perfect domination numbers of a tree. Mathematics 8 (2020) 966.

[19] I.G. Yero and A. Cabrera Martínez, A characterization of trees with equal Roman $\{2\}$-domination and Roman domination numbers. Commun. Comb. Optim. 4 (2019) 95-107.

\section{Subscribe to Open (S2O) A fair and sustainable open access model}

This journal is currently published in open access under a Subscribe-to-Open model (S2O). S2O is a transformative model that aims to move subscription journals to open access. Open access is the free, immediate, online availability of research articles combined with the rights to use these articles fully in the digital environment. We are thankful to our subscribers and sponsors for making it possible to publish this journal in open access, free of charge for authors.

\section{Please help to maintain this journal in open access!}

Check that your library subscribes to the journal, or make a personal donation to the S2O programme, by contacting subscribers@edpsciences.org

More information, including a list of sponsors and a financial transparency report, available at: https://www. edpsciences.org/en/maths-s2o-programme 